\title{
Advanced Plasma Propulsion for Human Missions to Jupiter
}

\author{
Benjamin B. Donahue', J Boise Pearson ${ }^{2}$ \\ 'Boeing Space Systems, 499 Boeing Blvd., Huntsville, AL 35824 \\ ${ }^{2}$ Propulsion Research Center/TD40, NASA Marshall Space Flight Center, AL 35812
}

\begin{abstract}
This paper will briefly identify a promising fusion plasma power source, which when coupled with a promising electric thruster technology would provide for an efficient interplanetary transfer craft suitable to a 4 year round trip mission to the Jovian system. An advanced, nearly radiation free Inertial Electrostatic Confinement scheme for containing fusion plasma was judged as offering potential for delivering the performance and operational benefits needed for such high energy human expedition missions, without requiring heavy superconducting magnets for containment of the fusion plasma. Once the Jovian transfer stage has matched the heliocentric velocity of Jupiter, the energy requirements for excursions to its outer satellites (Callisto, Ganymede and Europa) by smaller excursion craft are not prohibitive. The overall propulsion, power and thruster system is briefly described and a preliminary vehicle mass statement is presented.
\end{abstract}

\section{FUSION PROPULSION SYSTEMS}

Mission and system studies have shown that fusion reactors potentially may be the most attractive energy source for space power and propulsion systems. Multiple magnetic confinement concepts have been examined for mission applications. These systems offer very high thrust and specific impulse, but significant engineering implementation challenges exist that are largely inherent to magnetically confined plasma fusion systems whether terrestrial or space based. These include; further development of superconducting magnet technology, development of long-lived first surface material (That material next to the hot plasma), removal of heat flux from the first surface and the protective blanket for superconducting coils, development of a suitable blanket to shield superconducting magnets from the neutron flux, conversion of the plasma enthalpy to electrical power for onboard and propulsion system use, and radiation damage of wall materials by neutron flux.

Most of these challenges can only be met by adding significant amounts of weight to the propulsion system. An alternative fusion power concept exists that offers potential for high performance without the mass intensive engineering solutions to these challenges..

\section{Inertial Electrostatic Confinement}

A brief investigation was conducted of several fusion power and propulsion system concepts. An advanced fusion power source identified by its Inertial Electrostatic Confinement (IEC) scheme for containing the fusion plasma, was chosen for further consideration. It was judged as offering potential for delivering the performance and operational benefits needed for high energy human expedition missions to Jupiter. IEC reactor technology provides electrostatic heating and confinement of the fusion plasma, as well as direct energy conversion to electrical energy. It therefore does not require the heavy magnets, high-power drivers, thermodynamic electrical plant and other massive components required of thermal fusion propulsion devices typically analyzed in advanced propulsion studies (Miley, 1995).

These IEC fusion systems would enable a much lower weight for the propulsion system when compared to those fusion concepts requiring heavy magnetic systems for confinement of the plasma. Coupled with state-of-the-art magnetoplasmadynamic (MPD) electric thruster technology, this propulsive concept appears to offer an attractive solution for a fast transfer mission to Jupiter. 


\section{IEC System Description}

The term IEC describes a family of fusion reactor concepts in which concentric electrostatic fields are used to accelerate ions radially into the center, where the charged particles converge and react, yielding energetic charged fusion products (Bussard, 1995, Miley, 1995). A very high fusion power energy density (of roughly mono-energetic ions) is achieved as these ions converge at the center of the vessel, forming a dense central core region where fusion occurs. Fuel combinations of D-T, D-D, D- ${ }^{3} \mathrm{He}$ and $\mathrm{p}-{ }^{11} \mathrm{~B}$ are being investigated. The $\mathrm{D}-{ }^{3} \mathrm{He}$ and $\mathrm{p}-{ }^{-11} \mathrm{~B}$ reactions are aneutronic, eliminating or greatly reducing the need for neutron shielding. Rather than utilizing fusion energy to directly heat a propellant, a Direct Energy Converter (DEC) can be used to obtain electric power from the radially moving, positively charged fusion products as they escape the active region. This DEC is built by surrounding the electric confinement volume with an electrically biased grid system that decelerates these ions, where the ions lose energy to the electric field. The ions can be made to arrive at the collecting grids with little or no residual energy (Bussard, 1995, Post, 1969, Roth, 1970). The general feasibility of this means of direct conversion has been proven by work at the Lawrence Livermore National Laboratory in earlier (1973-83) research studies (Bussard, 1995). This DEC can produce megavolt dc current at levels required for the MPD thrusters. Specifics on the DEC, cooling system, power conversion and electric thrusters have been developed in some detail (Miley, 1993). Since the IEC is used as an electrical source, the fusion fuel need not be exhausted for propulsion, hence fuel recycle is possible.

\section{MPD THRUSTERS}

The MPD thruster dates back to the 1960s and is a promising candidate for interplanetary missions because it has the potential to run at multi-megawatt power levels and produce 100's of Newtons of thrust. By operating at high current levels, the MPD thruster can accommodate high input power levels without requiring complex power conditioning to boost voltage (Cassady, 1988). The inherent simplicity of the device, coupled with a high power handling capability makes it attractive for energetic missions. In a simple MPD thruster, a plasma current is generated by the arc between the tip of an axial conducting bar and a coaxial cylindrical wall. The current along the conducting bar creates an azimuthal magnetic field that interacts with the plasma current. The resulting Lorentz force has two components; a radially inward force that constricts the flow, and a driving force along the axis that produces the directed thrust. Since ions and electrons are produced and accelerated in bulk, the accelerated stream as a whole is neutral and unpolarized, (Demetriades, 1962). This is in contrast to the lon engine, wherein ions and electrons are produced, but only the ions are accelerated by electrostatic means. The neutral plasma exhaust of the MPD thruster eliminates the need for a separate neutralizer.

\section{MPD Thruster Demonstrations}

Both steady state and pulsed MPD systems have been developed. Megawatt pulsed MPD systems have demonstrated efficiencies above 50 percent with hydrogen propellant. Noble gas propellants can also be used, but with lower specific impulses. Princeton University recently presented results of lab tests with a Russian thruster. This thruster is a Lorentz Force Accelerator (a variant on MPD thrusters) which uses lithium propellant. Adding a little barium to the mix increases thruster lifetime to thousands of hours. The thruster has operated at $121 \mathrm{~kW}$, $44.2 \%$ efficiency, and $3568 \mathrm{sec}$ Isp. Their partners at Energia have run a similar unit a $500 \mathrm{~kW}$ input power with $12.5 \mathrm{~N}$ thrust for 500 hours (Choueiri, 1998). Other testing at Princeton University (Ziemer, 1998), the Jet Propulsion Laboratory (King, 1985) and at other private and government laboratories have characterized the performance of MPD thruster technology, including thrusters of the 70 Newton capability chosen for this study.

\section{Propulsion System For Demanding Missions}

The IEC / MPD thruster combination represents an attractive propulsion system for demanding, high delta velocity human exploration transfers to the outer planets. Though the IEC fusion technology would require a very significant technology development program, the interplanetary transfer capability enabled by it would be immensely more robust than the chemical, solar and nuclear fission concepts currently advocated for human Earth-Mars missions. 
The $12 \mathrm{MW}$ IEC fusion reactor chosen for this mission is taken from the literature (Miley, 1995). The MPD thruster chosen is within the range of demonstrated laboratory performance, and is used in a cluster of six thrusters. Table 1 lists characteristics of the IEC reactor (Miley, 1995), MPD thrusters and combined propulsion system chosen for the mission in this study.

TABLE 1. IEC / MPD Propulsion System Characteristics.

\begin{tabular}{|ll|ll|ll|}
\hline IEC Fusion Reactor & Single MPD Thruster & & Total Propulsion System \\
\hline Fusion power & $12 \mathrm{MW}$ & Thruster Isp & $3,000 \mathrm{~s}$ & Thrust (6 thrusters) & $408 \mathrm{~N}$ \\
Reactor mass & $5 \mathrm{mt}$ & Thruster efficiency & $50 \%$ & Isp & $3,000 \mathrm{~s}$ \\
Reactor diameter & $5.3 \mathrm{~m}$ & Thruster specific mass & $0.1 \mathrm{~kg} / \mathrm{kW}$ & Propellant flowrate & $.0138 \mathrm{~kg} / \mathrm{s}$ \\
Specific power & $2.4 \mathrm{~kW} / \mathrm{kg}$ & Thrust per thruster & $68 \mathrm{~N}$ & Thrust $/$ Weight & .008 \\
& & Propellant feed pressure & $1 \mathrm{~atm}$ & Specific jet power & $1.2 \mathrm{~kW} / \mathrm{kg}$ \\
& & Propellant & Krypton & & \\
& & Propellant flowrate & $0.0023 \mathrm{~kg} / \mathrm{s}$ & & \\
\end{tabular}

\section{MISSION DESCRIPTION}

A four year round trip trajectory to Jupiter was chosen for analysis. This direct Earth-Jupiter-Earth mission is characterized by an outbound leg of just over 2 years, a one month stay time, and a 2 year inbound leg. Transfer vehicle operations are described as follows: about one month before the Trans-Jovian Injection window opens, a test crew will board the vehicle for final tests and pre-orbital-launch checkout. One week before the window opens the mission crew will board the vehicle in LEO; after a tie-in period the test crew will return to Earth on the shuttle that delivered the mission crew. The vehicle departs low Earth orbit (LEO) and conducts a slow spiral out typical of low thrust electric propulsion vehicles. (Vehicle thrust to weight at departure is on the order of $10^{-4}$.) The spiral out continues until the vehicle nears Earth escape velocity, then commences on a heliocentric transfer trajectory to Jupiter, at 5.2 astronomical units (AU) from the Sun. After arrival and matching of Jovian velocity, a high altitude parking orbit is established, conducive to exploration of one of the outer satellites. Transfer and descent preparations begin, including high-resolution imagery and viewing of the planned landing sites. Several members of the crew enter the excursion craft and transfer to one of the Jovian satellites for a one month stay, while the remaining crew stays onboard the transfer vehicle directing the operations of unmanned probes sent to Jupiter and its other moons.

After the surface mission is accomplished, the crew returns to the orbiting transfer vehicle, jettisons the ascent stage and departs for Earth on a two year inbound trajectory. About 16 hours before Earth arrival, the crew enters the small Crew Return Vehicle (CRV). At entry minus 12 hours the CRV separates from the rest of the transfer stage. The transfer vehicle is to be expended, and thus is not on an Earth atmosphere intercept path, and the CRV makes a burn of about $10 \mathrm{~m} / \mathrm{sec}$ delta velocity to place it on its entry path. The transfer stage is expended as it swings by Earth, continuing on a long lived heliocentric orbit. The CRV enters Earth's atmosphere, decelerates, and either rendezvous with the space station, or deploys parachutes, and makes a landing to complete the mission.

\section{Jovian Destinations}

Due to its immense mass and gravity, a manned descent into, and return from Jupiter itself was deemed inadvisable considering the modest efficiencies of current high thrust propulsion systems. The mass of Jupiter is 340 times greater than the Earth. Even the mass benefit associated with utilization of in-situ resources for ascent was considered inadequate for returns from descents deep into the planet's atmosphere. The outer Jovian satellites are however, accessible. Once the transfer stage has matched the heliocentric velocity of Jupiter, or entered an appropriate high altitude parking orbit around it, the energy requirements for excursions to these satellites are not prohibitive. The actual excursion / lander craft would use traditional high thrust-to-weight $(\mathrm{t} / \mathrm{w})$ chemical propulsion, or fission nuclear thermal propulsion for ascent/descent propulsion (Donahue, 1995). These could provide the modest delta velocities $(2.1-2.8 \mathrm{~km} / \mathrm{s})$ needed for the near vicinity orbital transfers to one of Jupiter's outer satellites, such as Callisto, Ganymede or, perhaps most interesting, Europa. It is one of these to which future Jovian exploration missions are most likely to be targeted (Beebe, 1997). 


\section{Delta Velocity Requirements}

Mission trip times and delta velocity increments are provided in Table 2. Low-thrust departures from within a sizable gravity field are relatively inefficient when compared to high thrust, impulsive departures. For comparison, the delta velocities for a high thrust transfer vehicle flying a comparable 4 year round trip trajectory are also shown in Table 2.

TABLE 2. Jovian Mission Duration and $\triangle \mathrm{V} \mathrm{s}$.

\begin{tabular}{|lc|lrr|}
\hline Mission Leg & Months & Event & $\begin{array}{l}\text { Low-Thrust } \\
(\mathbf{m} / \mathbf{s})\end{array}$ & $\begin{array}{l}\text { High-Thrust } \\
(\mathbf{m} / \mathbf{s})\end{array}$ \\
\hline LEO to High Earth Orbit spiral & 3 & LEO to near escape velocity & 6118 & 2600 \\
High Earth Orbit to Jupiter & 24 & Trans Jupiter injection & 20280 & 8000 \\
at Jupiter & 1 & Jovian injection & 13520 & 5500 \\
Jupiter to Earth flyby & 24 & Trans Earth departure & 13520 & 5500 \\
& & Earth arrival burn (aerobrake) & 10 & 10 \\
\hline Total & 51 & Totals & 53448 & 21610 \\
\hline
\end{tabular}

\section{JOVIAN TRANSFER VEHICLE}

The Jovian transfer vehicle (JTV) consists of the IEC fusion power system and MPD thrusters at one end and the crew habit and lander excursion craft at the other end, separated by a truss section with attached propellant tanks. Though hydrogen would produce the highest specific impulse (Isp), xenon or krypton would be used as propellant due to boiloff concerns with hydrogen. Krypton and xenon are inert, and neither will explode or corrode fuel lines or tanks. At typical storage pressures xenon is about 60 percent denser than water, thus the tankage fraction could be considerably less than it would be if hydrogen were utilized.

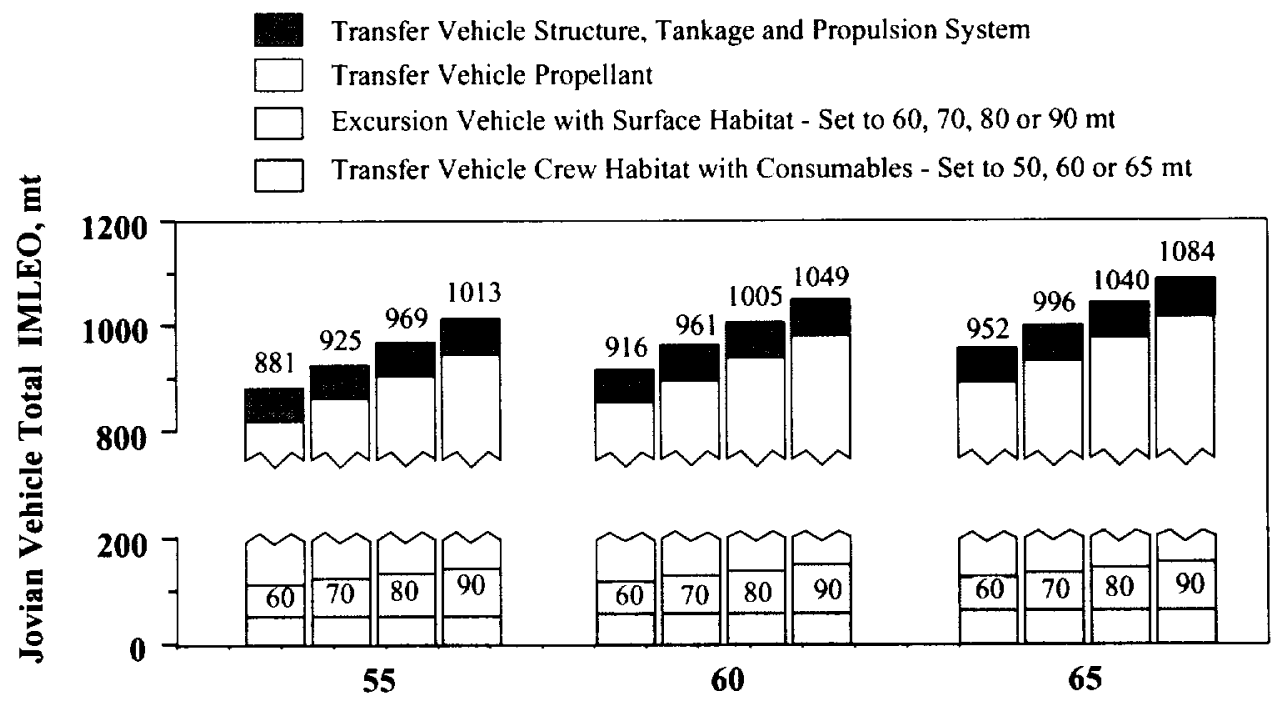

Crew Habitat Mass and Consumables for Transfer Vehicle, mt

FIGURE 1. Human Jovian Mission Vehicle Mass Estimates

Jovian vehicle Initial Mass in Low Earth Orbit (IMLEO) values were calculated for several values of excursion vehicle masses (lander) and transfer vehicle crew habitat masses (Donahue, 1998). Excursion vehicle masses of 60, 70,80 , and $90 \mathrm{mt}$ were utilized. This mass includes the ascent stage, the descent burn tankage, the crew surface habitat, the surface exploration equipment and rovers. JTV crew habitat masses (including consumables) of 55, 60, and $65 \mathrm{mt}$ were utilized. The habitat mass will determine the number of crew the vehicle can accommodate. The crew has four separate habitable volumes that can serve as living, working and recreational space on the outbound 
journey; (1) the large transfer vehicle habitat, the lander's (2) surface habitat and (3) small ascent crew cab, and the (4) CRV capsule. On the return leg only the large habitat and the CRV volumes would be available. IMLEO estimates are shown in Figure 1. The relative proportions of the propellant mass and inert vehicle mass (structure, propulsion and tankage) are given for the 12 combinations of habitat and lander masses. A propellant reserve allotment of 1 percent for each burn was allocated. As a weight saving measure, the Trans-Jupiter injection propellant tank is jettisoned after it is emptied.

\section{CONCLUSIONS}

The combination of an IEC nuclear fusion power source and a cluster of high power MPD thrusters represents a mass efficient propulsion system capable of providing the high delta velocities required for fast trip human exploration missions to Jupiter. The tasks needed to bring such a system up to the technology level necessary for inspace operation principally involve the development and testing of electrostatic confinement of fusion plasmas and direct electrical energy production characteristics of the selected IEC system. Larger scale follow on tests would be necessary to demonstrate the systems capability for multiyear power production at multimegawatt power levels. Jovian satellite excursion vehicles could utilize traditional chemical propulsion or nuclear thermal propulsion systems for their less energetic landing and ascent maneuvers.

\section{ACKNOWLEDGMENTS}

This paper is largely based on work done in support of the Affordable In-Space Transportation (AIST) concept definition study funded by the NASA Marshall Space flight Center and supported by Boeing (contract NAS8-5000 Schedule F, TOF-028).

\section{REFERENCES}

Beebe, Reta, Jupiter, the Giant Planet, Smithsonian Institution Press, 1997

Bussard, R. W. and Jameson, L. W., "Inertial-Electrostatic-Fusion Propulsion Spectrum: Air-Breathing to Interstellar Flight," Journal of Propulsion and Power, Vol. 11, No. 2, March-April 1995.

Cassady, R. J., "An MPD Thruster Driven Cargo Ferry for Support of the Manned Mars Mission," AIAA Paper 88-2896, July 1988

Choueiri, Edgar, Princeton University, Presentation to Ninth Annual NASA-JPL Advanced Propulsion Research Workshop, Jet Propulsion Lab, March 11-13, 1998.

Demetriades, S. T., Plasma Propulsion Technical Memorandum NSL 62-14, Northrop Space Laboratories, Jan 1962

Donahue, B. B., Editor, Affordable In-Space Transportation Concepts, Special Studies: Advanced Plasma Propulsion Applications to Human Missions to Jupiter, Contract NAS8-50000, Schedule F, TOF-028, Task 3 Final Report, Boeing Space Systems, Huntsville, Alabama, January 1998.

Donahue, B. B., "Mars Ascent-Stage Design Utilizing Nuclear Propulsion," Journal of Spacecraft and Rockets, Vol. 32, No. 3, May-June 1995.

Donahue, B. B., "Self Assembling Mars Transfer Vehicles: The Preferred Concept of the Space Transfer concepts and Analysis for Explorations Missions Study," AIAA paper 94-2761, July 1994

Donahue, B. B., Editor, Space Transfer Concepts and Analyses for Exploration Missions, Contract report, Nuclear Thermal Propulsion Implementation Plan and Element Description Document, NAS8-37857, Document No. D615-10069, Boeing Civil Space Product Development Group, Huntsville, Alabama, Sept. 1993.

King, D. Q., "Feasibility of Steady-State, Multi-Megawatt MPD Thruster," AIAA Paper 85-2004, 1985.

Miley, G. H., Burton, R. L., Javedani, J., Gu, Y., Satsangi, A., Heck, P., Nebel, R. A., and Schulze, N., "Inertial Electrostatic Confinement as a Power Source for Electric Propulsion," Vision-2I Conference Proceedings, NASA Conference Publication. 10129, 1993

Miley, G. H., Satsangi, A. J., DeMora, J., Javedani, J. B., Gu, Y., Burton, R. L. and Nakashima, H., "Innovative Technology for an Inertial Electrostatic Confinement Fusion Propulsion Unit,". Fusion Energy in Space Propulsion, Vol. 167, Progress in Astronautics and Aeronautics, 1995

Post, R. F., "Direct Conversion of Thermal Energy of High Temperature Plasma," Bulletin of American Physics Society, Vol. 14, No. 11. November 1969.

Roth, J. R., Rayle, W. D., and Reinmann, J. J., "Technological Problems Anticipated in the Application of Fusion Reactors to Space Propulsion and Power Generation," NASA TM X-2106, NASA Washington, D. C. October 1970. 
Ziemer, John K., Private communication, Princeton University, March 1998 